\title{
Band splitting and relative spin alignment in bilayer systems.
}

\author{
A.A. Ovchinnikov ${ }^{1)}$ and M.Ya. Ovchinnikova ${ }^{2)}$. \\ ${ }^{1,2)}$ Joint Institute of Chemical Physics, RAS, Moscow. \\ 1) Max Planck Institute for Physics of Complex Systems, Dresden.
}

\begin{abstract}
Influence of relative spin alignment on the band splitting and magnetic excitations in bilayer correlated systems is studied. Splitting occurs to be large or small depending on relative orientation of staggered spins of the layers. Change of the ground state spin configuration with doping is shown. Behavior of bilayer splitting in $\mathrm{Bi}_{2} \mathrm{Sr}_{2} \mathrm{CaCu}_{2} \mathrm{O}_{8+\delta}$ allows to suppose that superconducting transition is accompanied by transformation of spin configuration of system.
\end{abstract}

PACS: 71.10.Fd, 71.27.+a, 71.10.Hf

One of properties of high $T_{c}$ superconductors - a dependence of their characteristics on number of the $\mathrm{CuO}_{2}$ layers - shows importance of interlayer coupling in them. In recent photoemission experiments [1, 2, 3] the bilayer splitting of the bands and the Fermi surfaces (FS) in $\mathrm{Bi}_{2} \mathrm{Sr}_{2} \mathrm{CaCu}_{2} \mathrm{O}_{8+\delta}$ (BSCCO) have been observed. Unusual observation was a significant decrease in bilayer splitting at $k \sim(\pi, 0)$ in course of the superconducting (SC) transition [2]. Another phenomena which is observed in the bilayer (but not monolayer) cuprates is the appearance in SC state of a spin resonance in inelastic neutron scattering [ [6, 5, 6]. Explanation of these phenomena is important for understanding the electronic structure and mechanisms of HTSC displaying many properties of doped Mott insulator. The main feature of latter is the Hubbard splitting of band induced by antiferromagnet $(\mathrm{AF})$ fluctuations. Though the long range AF order disappear at small doping $\delta_{c}=1-n_{c} \sim 0.05$, the local 2D spin order probably retains in sufficiently large doping range including the range of superconductivity. Among arguments in favour of such local AF order there are data on $\mu \mathrm{SR}, \mathrm{NMR}, \mathrm{NQR}$ [7, 8], large length of spin correlations with $Q=(\pi, \pi)$ and smooth evolution of collective magnetic excitations with doping (see 
review [■] ) and others. Finally, recently a direct proof of the AF order even in SC state of $\mathrm{YBa}_{2} \mathrm{Cu}_{3} \mathrm{O}_{6.5}$ have been obtained [9, 10] in nanosecond scale.

Detailed analysis of the AF bands and interactions of the Hubbard model have been done in classic work [11]. Simple mean field method gives the overestimated values $d_{0}$ of staggered spin and of the doping boundary $\delta_{c} \sim 0.45$ of a range of the AF order. The band renormalization on base of the zero AF approximation [11] or the calculations of t-t'-U Hubbard model using the slave-boson technique [12] or the states with the valence bond correlations [13] - all these methods give the smaller values of $d_{0}$ and of the boundary doping $\delta_{c} \sim 0.3$ at which the local magnetization disappears. According [13] the range $\delta<\delta_{c}$ includes entirely the range of superconductivity. Retaining of the Hubbard splitting of band at $\delta<\delta_{c}$ in the t-t'-U [13] and t-t'-J [14, 15, 16] models implies the change of the FS topology at the optimal doping with transition from the hole pockets to large FS.

If such picture is true then interactions between the layers in bilayer systems may significantly depend on relative orientation (alignment) of local staggered spins of the layers. It must be so even if the spin orientation is not stationary in strong sense, but is retained only on some time interval. In case of small difference in the energies of various configuration of spin alignment one can expect a strong influence of doping and temperature on the properties of magnetic excitations in system. A search of such effects may help to elucidate the origin of the magnetic resonance in neutron scattering in $\mathrm{YBCO}$, BSCCO.

Present work summarizes the results of variational calculations of bands, full energies, phase curves $T_{c}(\delta)$ and the band splittings in the bilayer t-t'-U Hubbard model. Calculations are made on base of the correlated states with the valence bond (VB) formation - the band analog of the RVB state of Anderson. Previously [13 it was shown that the correlated hopping interaction appearing in the effective Hamiltonian during the VB formation does provide the hole attraction in d- channel and corresponding dsuperconductivity compatible with AF spin order. Here we use the same method for study the effects of interactions of two layers. The questions of interest are: Is the large doping range of local AF order retained? If so, then what are the relative alignment of spin systems of both layers and the bilayer splitting at various doping?

A difference between given approach and the study [17] of bilayer Hubbard model by the FLEX (fluctuation exchange) method must be stressed. According FLEX the hole 
attraction is provided by long range spin fluctuations. In our approach the short range AF correlations are decisive. In simpliest variant they correspond to the VB formation, i.e. formation of singlets on the bonds of the nearest centers. Unlike the averaged consideration in FLEX we make the calculations for two specific spin configurations of bilayer system - homogeneous states with the same or opposite alignment of staggered spins of both layers. We denote them as the $F_{z}$ or $A F_{z}$ (ferro- or antiferro-magnet over $\mathrm{z}$ direction) alignment correspondingly, though both cases refer to local AF spin order in each layer. We consider only homogeneous states, though many neutron experiments give indication on the stripe-type or spiral incommensurate structures in cuprates. Nevertheless the effects discovered in our calculations for two homogeneous spin structures must undoubtedly be taken into account in discussing the observed bilayer splitting and magnetic properties of cuprates.

We start from the Hubbard Hamiltonian of bilaeyr system $H\left(U, t, t^{\prime}, t_{z}\right)$. The main intralayer interactions are determined by standard parameters $U, t$ of Hubbard model. Additional interactions include the hoppings $t^{\prime}, t_{z}$ between the next nearest centers inside the layers and between the cites of different layers. Positive signs of standard parameters of strong coupling $t, t^{\prime}, t_{z}$ are defined in such way that the zero bands (at $U=0$ ) have a form

$$
\epsilon_{ \pm}^{(0)}=-2 t\left(\cos _{x}+\cos _{y}\right)+4 t^{\prime} \cos _{x} \cos k_{y} \mp \frac{1}{4} t_{z}\left(\cos k_{x}-\cos k_{y}\right)^{2}
$$

Expression for splitting $\Delta \epsilon(k)=\epsilon_{+}-\epsilon_{-}$between the bonding and antibonding zero bands is derived in [17].

A variational correlated state $\Psi=\hat{W}(\alpha) \Phi$ with correlations of the VB type is constructed [13] as unitary transformed uncorrelated state $\Phi$

$$
\hat{W}(\alpha)=\exp [\alpha Z] ; \quad Z=\frac{1}{2} \sum_{\sigma,<n m>, \gamma}\left(c_{\gamma n \sigma}^{\dagger} c_{\gamma m \sigma}-h . c .\right)\left(n_{\gamma n,-\sigma}-n_{\gamma m,-\sigma}\right) ;
$$

Here $\gamma=0,1$ refer to layers a,b. A choice of unitary operator $W(\alpha)$ with variational parameter $\alpha$ is explained in [13]. Variational treatment of problem with Hamiltonian $H$ in basis of correlated state $\Psi$ is equivalent to treatment of the effective Hamiltonian $H_{\text {eff }}(\alpha)=W^{\dagger}(\alpha) H W(\alpha) \approx H+\alpha[H, Z]+\frac{\alpha^{2}}{2}[[H, Z]$ in mean field approximation. We use the most general uncorrelated states $\{\Phi\}$ of the BCS type with anomalous averages of d-symmetry and the AF spin order. The mean energy over such state $<H>_{\Psi}=<H_{e f f}>_{\Phi}=\bar{H}\left(y_{\gamma \nu}, z\right)$ is obtained as an explicit function of a set of one- 
particle averages $\left\{y_{\nu}, z\right\}$ over uncorrelated state $\Phi$. The set of these averages contains the density components $r_{\gamma l}=<c_{\gamma n \sigma}^{\dagger} c_{\gamma, n+l, \sigma}>$, analogous components of staggered spin $d_{\gamma l}=(-1)^{n} \frac{\sigma}{|\sigma|}<c_{\gamma n \sigma}^{\dagger} c_{\gamma, n+l, \sigma}>$, anamalous averages $w_{\gamma l}=\left(l_{x}^{2}-l_{y}^{2}\right)<c_{\gamma n \sigma} c_{\gamma, n+l, \sigma}>$ of d-symmetry for each layer $\gamma=0,1$ and a value $z=t_{z}^{-1}<T_{z}>$ determining the average interlayer hopping. For spin components we study two variants of relative alignment $d_{\gamma=1, l}=\zeta_{d} d_{\gamma=0, l}, \quad \zeta_{d}= \pm 1$. Selfconsistent procedure of the energy minimization over $\Phi$ and of subsequent minimization over $\alpha$ is standard one [13]. Details of calculations will be presented elsewhere.

For main parameter we use a value $U / t=8$ following from cluster derivation of single band model. Parameter $t^{\prime}$ owing to its contribution into the band dispertion regulates the position $E_{V H S}=E(\pi, 0)$ of the van-Hove singularity (VHS) in the density of state relative to the edge of the lower Hubbard band. In the monolayer $t-t^{\prime}-U$ or $t-t^{\prime}-J$ models a value of $t^{\prime}$ is directly connected with the optimal doping $\delta_{\text {opt }}$, since a maximum of $T_{c}$ corresponds to coincidence of the chemical potential $\mu$ with $E_{V H S}$. According the models [13, 14, 15, 16] a resonable value of $\delta_{\text {opt }} \sim 0.2 \div 0.25$ corresponds to values $t^{\prime} / t \sim 0.05 \div 0.1$. These values are less than $t^{\prime} / t \sim 0.2 \div 0.4$ obtained from fitting of photoemission data and the band calculation data on base of the strong coupling scheme. Here we vary parameter $t^{\prime}$ in limits $t^{\prime}=0.05 \div 0.3$. Parameter of interlayer hopping is also varied as $t_{z} / t=0.07 \div 0.3$. Previous calculations without renormalization gave $t_{z} / t \sim 1 / 3$ [17]. Recent measurements of bilayer splitting $\Delta=2 t_{z}=\delta \epsilon_{k}(\pi, 0)$ [1, 2] give the value $t_{z} / t \leq 0.1$

Fig. 1 presents typical doping dependencies of spin density $d_{0}$, transition temperature $T_{c}(\delta)$ and a difference $\Delta H=\bar{H}\left(A F_{z}\right)-\bar{H}\left(F_{z}\right)$ of average energies of bilayer system with two types of relative alignment of the layer's spins. Staggered spin density $d_{0}$ has nonzero value at wide range of doping including the region of supercondutivity. Quantity $\Delta H(\delta)$ changes its sign at some doping. In the undoped system, $\delta=0$, the configuration $A F_{z}$ with opposite alignment of the layer's spins occurs to be lower than the $F_{z}$ configuration. At large doping $\delta$ one have inverse situation up to boundary value $\delta_{c}$, at which the local magnitization disappears. Negative sign of $\Delta H$ at $\delta=0$ is explained by a positive exchange constant $J_{a b} \sim 2 t_{z}^{2} / U>0$. Change of sign of $\Delta H$ at some $\delta$ is a result of the bilayer splitting of the bonding and antibonding bands, in particular, the splitting of VHS, and different occcupancy of them for two types of spin alignment. Maximum of $\Delta H(\delta)$ 
corresponds to optimal doping $\delta_{\text {opt }}$ for the parent monolayer model. At this doping one has $E_{V H S}^{1 L}-\mu=0$. Increase of $t^{\prime}$ shifts both the $\delta_{\text {opt }}$ and the position of maximum of $\Delta H(\delta)$. Such relation is not occasional.

According [17] the bilayer splitting $\delta \epsilon_{z}^{(0)}=2 t_{z}\left(\cos k_{x}-\cos k_{y}\right)^{2} / 4$ of the zero bands has a maximum in region $k \sim(\pi, 0)$ forming the VHS in density of state. However, the splitting contributes to the average energy only if at this $k$ bonding and antibonding bands appear to be occupied and unoccupied correspondingly. The latter takes place when $E^{1 L}(\pi, 0)=E_{V H S}^{1 L}=\mu$ for the unsplit bands of the monolayer model. It remains to explain why a bilayer splitting manifests itself in the energy difference $\Delta H=\bar{H}\left(A F_{z}\right)-\bar{H}\left(F_{z}\right)$. The reason is in different bilayer splitting for different spin configurations.

Fig. 2 presents the density of state of lower Hubbard band for two configurations of spin alignment for system with small values $t^{\prime}, t_{z}$. Bilayer splitting is absent at $A F_{z}$ alignment, but sharply seen in $F_{z}$ configuration. This is due to different behaviour of matrix elements of the interlayer hopping between the states of lower Hubbard band of each layer. At $A F_{z}$ alignment these matrix elements disappear on the nesting lines. Different behaviour of DOS reflects itself directly on the form of the phase curves $T_{c}(\delta)$ for SC transition (Fig. 1). In case of $A F_{z}$ or $F_{z}$ configuration the curve $T_{c}(\delta)$ has one or two maxima correspondingly.

Thus the models with small $t^{\prime}, t_{z} \lesssim 0.1$ are characterised by 1) large $T_{c}^{\max } \sim 0.02 t \sim$ $116 K$ at $A F_{z}$ configuration; 2) close energies of both configurations, 3) small condensation energy; 4) lower $F_{z}$ configuration at $\delta \sim \delta_{\text {opt }} ; 5$ ) large (or zero) bilayer splitting in $F_{z}$ (or $A F_{z}$ ) configuration.

Unlike the normal state, a prediction of a lower spin configuration in the SC state is hardly possible. An estimate of the condensation energy and the step in a heat capacity occurs to be less than that observed in YBCO approximately in 5 times. For this reason we dicuss the spin configuration of SC state on base of the observed behaviour of bilayer splitting in BSCCO [1, []. This splitting decreases from $\delta \epsilon \sim 80 \mathrm{meV}$ in normal state down to $\delta \epsilon \sim 20 \mathrm{meV}$ in SC state. Such behaviour might be explained if we suppose that a transformation of configuration $F_{z} \rightarrow A F_{z}$ proceeds simultaneously with SC transition. One might suppose also that the SC transition as such is induced by the change of configuration, since such transition is accompanied by increase of the density of states at the Fermi level. 
For models with large values $t^{\prime}, t_{z}$ the types of dependencies $\Delta H(\delta), d_{0}(\delta)$ are retained. Increase of $t_{z}$ leads to increase of $|\Delta H|$ and the doping of the $F_{z}-A F_{z}$ crossover. Calculations confirm the wide doping range of AF local spin order for these model also. Value of $d_{0}$ is greater by an order of magnitude than the value of AF spin density observed in $\mathrm{SC}$ state of YBCO [9, 10] from elastic neutron scattering. The difference, possibly, is connected with large distribution of the spin directions (or signs) of different biplaines in crystal. Significant decrease of $T_{c}$ and a deformation of the phase curves with increase of $t^{\prime}, t_{z}$ are connected with decrease of the density of state on the FS for these models.

Influence of the spin alignment on the magnetic excitation spectrum is of interest in connection with discovery of the spin resonance in neutron scattering in SC state of cuprates and its unusual dispersion [10]. A widely discussed hypothesis [18 connects its origin with the so called $\pi$-resonance - an excitation of $e-e$ pair with $Q \sim(\pi, \pi)$ in triplet state. But the ratio of weights of such resonance to the integral (over $\omega$ ) intensity of the non-resonance magnetic excitations would be too small. Expected order of magnitude of this ratio is $\sim\left(w / d_{0}\right)^{2} \sim 0.01$. Interpretation of incommensurate patterns in the spin susceptibility on base of the nesting properties of FS also cannot describe a large intensity of spin excitations and their similarity in both the undoped dielectric materials and the doped metallic ones. The most probable hypothesis 19 implies the common magnetic origin of spin resonance and of the incommensurate anomalies of spin susceptibility $\chi^{\prime \prime}(q, \omega)$ in cuprates. Both features are explained by existence of the AF domains or the incommensurate modulation of the local staggered magnetization. The stripe-type structures have been observed in $\mathrm{LaSrCuO}$ and they may also present in the bilayer cuprates. But monolayer models [19] cannot explain the fact that the resonance appears only in superconducting state and only in odd channel of bilayer system. In this connection it is important to study the influence of spin alignment on magnetic excitations in bilayer systems.

Here we study such effects using a simpliest example of homogeneous model for "constructing" the spin resonace. Consider the fenomenological spin Hamiltonian with anisotropic interlayer interaction

$$
H=\sum_{\gamma=a, b} J_{0} \sum_{<n m>} \mathbf{S}_{n}^{\gamma} \mathbf{S}_{m}^{\gamma}+\sum_{n}\left\{J_{z, a b} S_{z n}^{a} S_{z n}^{b}+J_{\perp, a b}\left[S_{x n}^{a} S_{x n}^{b}+S_{y n}^{a} S_{y n}^{b}\right]\right\}
$$

The " $z$ "-direction is selected by vector of local staggered spins $\left.<\mathbf{S}_{n}^{\gamma}\right\rangle=\mathbf{e}_{z}\left(\zeta_{d}\right)^{\gamma} d_{0}$ of each layer $\left(\gamma=0,1\right.$ for layers a,b) with definite alignment $\zeta_{d}=1$ or -1 , stabilized by 
the nonspin interactions. Then the RPA consideration in the linear spin wave theory [20] gives the following frequencies of collective spin excitations for both types of alignment

$$
\begin{array}{lll}
F_{z}: & \omega^{\text {even }}(q) \approx 2 \Omega \sqrt{\varphi-g_{1}}, & \omega^{\text {odd }}(q) \approx 2 \Omega \sqrt{\varphi-g_{2}} \\
A F_{z}: & \omega^{\text {even }}(q) \approx 2 \Omega \sqrt{\varphi+g_{2}}, & \omega^{\text {odd }}(q) \approx 2 \Omega \sqrt{\varphi+g_{1}}
\end{array}
$$

Here $\varphi=2+\cos q_{x}+\cos q_{y}, \Omega=2 J_{0} d_{0}, g_{1(2)}=\left(J_{z, a b} \mp J_{\perp, a b}\right) / 2 J_{0}$.

Fig. 3 presents a dispersion of the spin excitations in even and odd channels for both configurations of a spin alignment for model with parameters $g_{1}=0.05, g_{2}=0.15$. Consider them in light of the above suggestion about simultaneous transformation $F_{z} \rightarrow$ $A F_{z}$ of the spin configuration and superconducting transition in bilayer cuprates. If it is true for our spin model (3), then at $T<T_{c}$ the intense peak appears in the odd channel of $\chi^{\text {odd }}$ at $q \sim(\pi, \pi)$ with the frequency $\omega=\Delta^{\text {odd }}=2 \Omega \sqrt{g_{1}}$, equal to a gap in the excitation spectrum in this channel for $A F_{z}$ spin configuration. This peak is absent in case of $F_{z}$ configuration which is supposed to be ground state configuration at $T>T_{c}$. Note that for $F_{z}$ configuration the low frequency excitations in $\chi^{\prime \prime}(q, \omega \rightarrow 0)$ correspond to momenta $q$ distributed along the circle $|\mathbf{q}-\mathbf{Q}|=\sqrt{2 g_{1}}$ instead of the discrete incommensurate momenta $Q_{\eta}=(\pi(1+\eta), \pi)$, observed for the low frequency excitations in YBCO [13]. They imply an existence of the incommensurate spin structures. Fig. 3c schematically presents the branches of the spin excitations for the spiral states or the states with modulation of a local spin $\left\langle S_{z n}>=d_{0} \cos \mathbf{Q}_{\eta} \mathbf{n}\right.$ of the monolayer (1L) model [20]. In [20] the resonance frequency have been identified as a frequency at the crossing point of branches at $q=(\pi, \pi)$.

Thus, it is confirmed that local magnitization $d_{0}$ is retained in large doping range for wide diapason of parameters $t^{\prime}, t_{z}$. The crossover of two spin configurations of the bilayer system proceeds at some doping. The lower normal state is that with the $A F_{z}$ or $F_{z}$ spin alignment at small or large doping correspondingly. Maximum of the energy difference of these configurations corresponds to the optimal doping of the parent monolayer system and is connected with the maximum splitting of van-Hove singularities. The bilayer splitting is zero or small for the $A F_{z}$ configuration, but large for the $F_{z}$ spin alignment. The observed in BSCCO large bilayer splitting in normal state, but small splitting in SC state [2] may indicate according our models that the transformation $F_{z} \rightarrow A F_{z}$ of configuration proceeds simutaneously with supercunducting transition. It is shown that the magnetic excitation spectrum depends dramatically on type of a spin configuration. 
The model spin system is presented for which a change of configuration $F_{z} \rightarrow A F_{z}$ is accompanied by appearance of resonance (or spin gap) in $\chi^{\text {odd }}$ at $q=(\pi, \pi)$.

A check of the above hypothesis requires further study, in particular calculations of magnetic excitations in case of the inhomogeneous (of the stripe type) spin structures of bilayer systems.

Work is supported by Russian Fund of Fundamental Reasearch (Projects No. 00-0332981 and No. 00-15-97334. Athours thanks V.Ya Krivnov for useful discussions and P.Fulde for possibility to work in Max Planck Institute for Physics of Complex Systems, Dresden.

\section{References}

[1] Y.-D.Chuan, A.D.Gromko, A.Fedorov et al. E-print archiv cond-mat/0102386

[2] D.L.Feng, N.P.Armitage, D.H.Lu et al. E-print archiv cond-mat/0102385

[3] J.Mesot, M.Boehm, M.R.Norman et al. E-print archiv cond-mat/0102339

[4] P.Bourges in "The gap Symmetry and Fluctuations in High Temperature Superconductors", ed. J.Bok, G.Deutscher, D.Pavuna, S.A.Wolf (Plenum Press).

[5] P.Bourges, Y.Sidis, H.F.Fong et al. Science 288, 1234 (2000).

[6] H.A.Mook, P.Dai, F.Dogan, R.D.Hunt et al. Nature 404 , 729 (2000).

[7] A.Rigamonti, F.Borza, P.Carett, Rep.Progr.Phys. 61, 1367 (1998).

[8] Ch.Niedermayer, C. Bernhard, T.Blasius et al. Phys.Rev.Lett. 80, 3843 (1998).

[9] H.A.Mook, P.Dai, F.Dogan, R.D.Hunt et al. E-print archiv cond-mat/0102047.

[10] Y.Sidis, C.Ulrich, P.Bourges et al. Phys.Rev.Lett. 864105 (2001).

[11] J.R.Schrieffer, X.G.Wen, F.C.Zhang, Phys.Rev. B 39, 11663 (1989).

[12] U.Trapper, D.Ihle, H.Fenke, Phys.Rev. B 52, 11553 (1995). 
[13] A.A.Ovchinnikov,

M.Ya.Ovchinnikova,

Phys.Lett.

A249, 531 (1998). A.A.Ovchinnikov, M.Ya.Ovchinnikova, E.A.Plekhanov, JETP Letters,67, 350 (1998); JETP 114, 985 (1998); 115, 649 (1999)].

[14] N.M.Plakida, V.S.Oudovenko, R.Horsch, and A.J.Liechtenstein, Phys.Rev. B55, 11997 (1997).

[15] R.O.Kuzian, R.Hayn, A.F.Barabanov, and L.A.Maksimov, Phys.Rev. B 58, 6194 (1998).

[16] F.Onufrieva, P.Pfeuty, and M.Kisilev, Phys.Rev.Lett B 82, 2370 (1999).

[17] A.I.Liechtenstein, O.Gunnarsson, O.K.Andersen, R.M.Martin Phys.Rev. B 54, 12505 (1996).

[18] E.Demler, S.C.Zhang Phys.Rev.Lett. 75, 4126 (1995).

[19] C.D.Batista, G.Ortitz and A.V.Balatsky, E-print archiv cond-mat/0008345.

[20] T.Ziman, P.-A.Lindgard, Phys.Rev. B 33, 1976 (1986).

\section{Captions to Figures}

Fig. 1. Doping dependence of the local magnitization $d_{0}(\delta)$ (top), of the $T_{c}(\delta)$ (middle) and value $w_{1}(\delta)$ of the anamalous order parameter (bottom) for configurations $A F_{z} F_{z}$ (curves 1 and 2 correspondingly). Curve 3 is the difference of energies of these configurations. All values $T_{c}, t_{z}, t^{\prime}, \Delta H$ are in unit $t$.

Fig. 2. Density of state for two types $A F_{z}$ and $F_{z}$ of spin alignment for models with small $t_{z}, t^{\prime}$. Thin solid and dashed lines refer to contributions in DOS from bonding and antibonding bands. The calculated DOS is smoothed with gaussian function with dispersion $\delta E=0.02 t, \mathrm{E}$ is in unit $t$.

Fig. 3. Changes in the magnetic excitations branches $\omega(q)$ during transformation $F_{z} \rightarrow A F_{z}$ of spin configuration of model (5) with anisotropic interlayer interaction with parameters $g_{1}=0.05, g_{2}=0.15$. A right figure is schematic presentation of the spin excitations in case of the spiral or modulated incommesurate spin structure with $Q_{\eta}=$ $(\pi(1-\eta), \pi), \eta=0.1$. Squares mark the supposed resonance frequencies in various interpretations. 


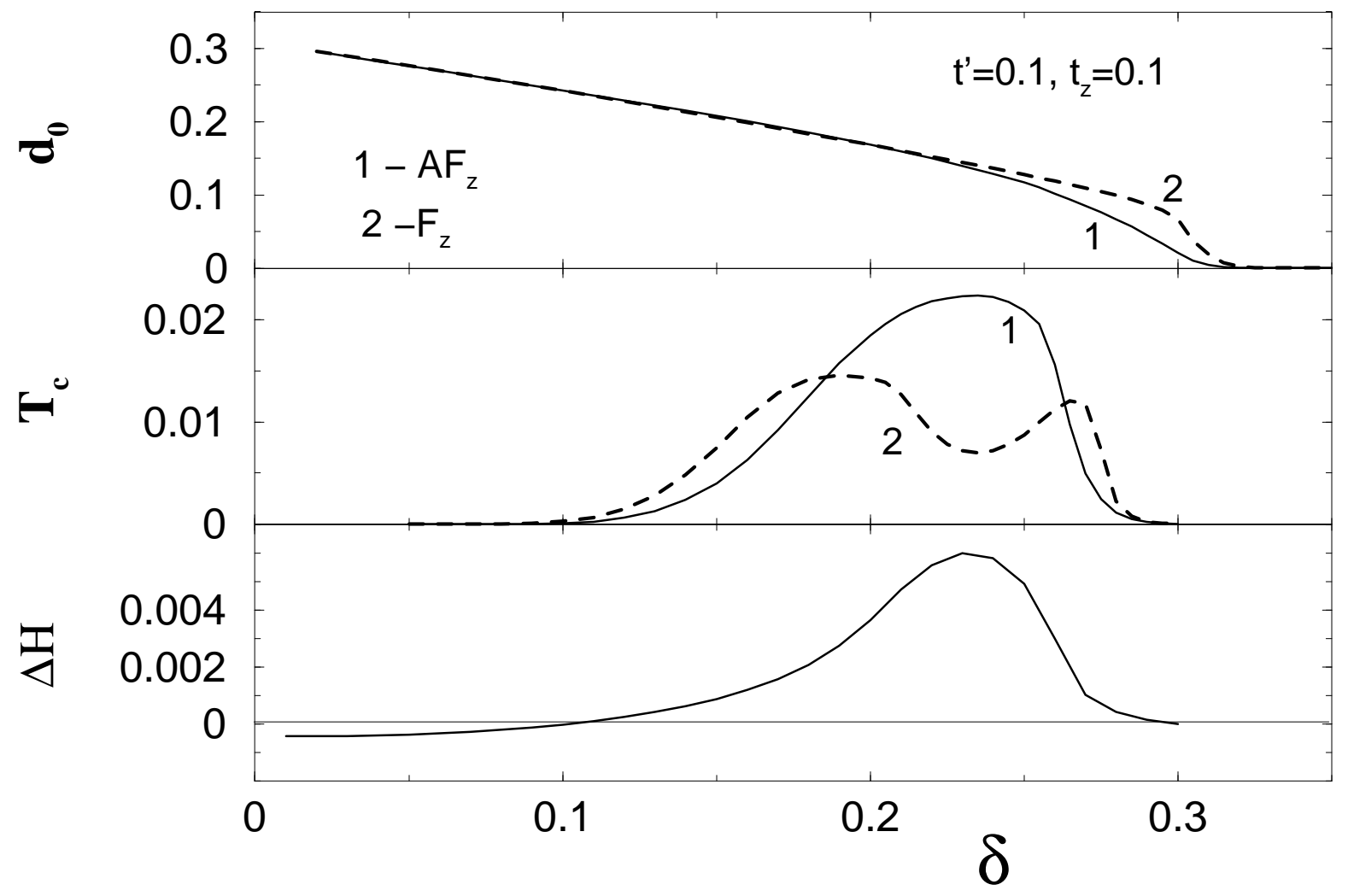




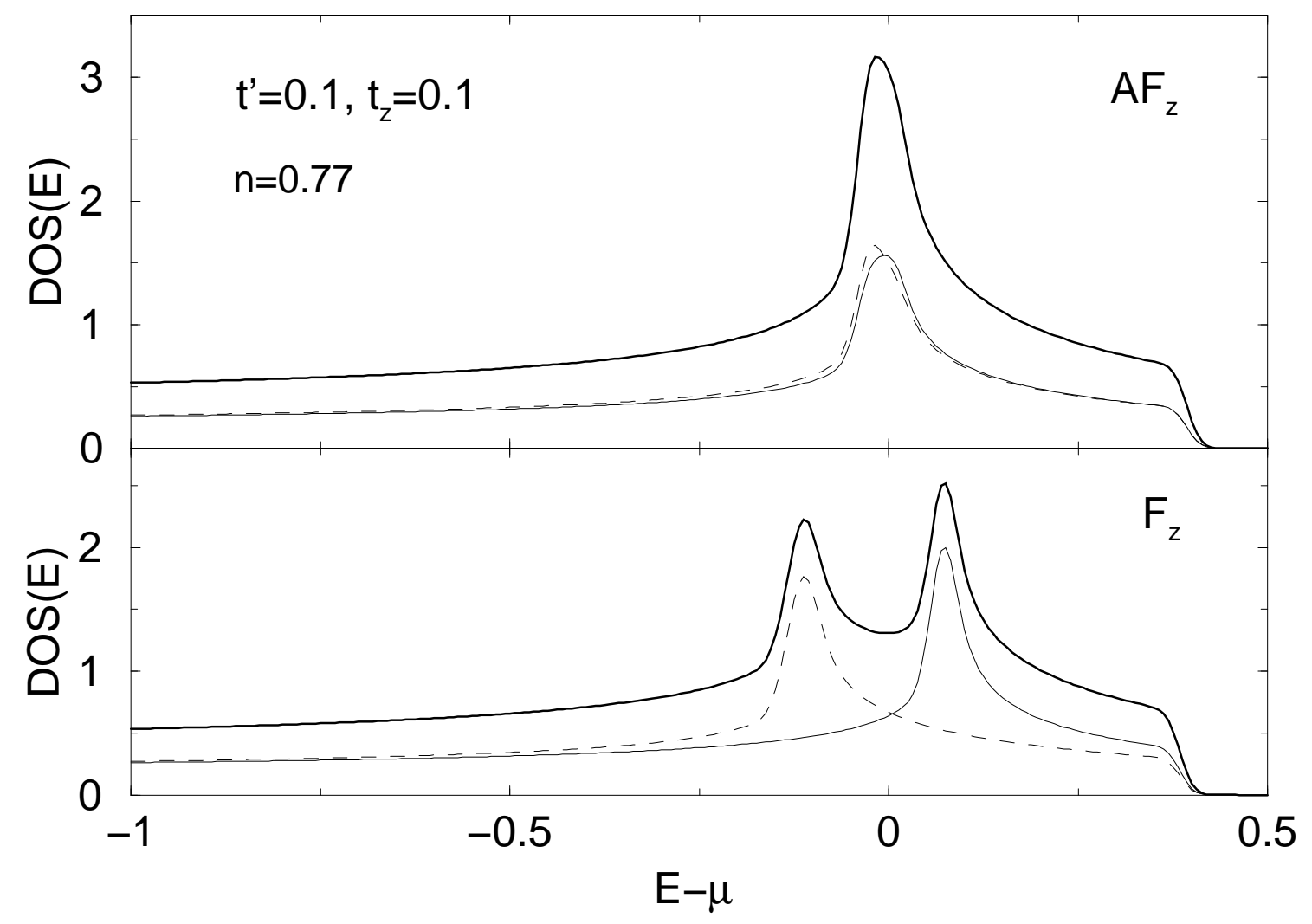




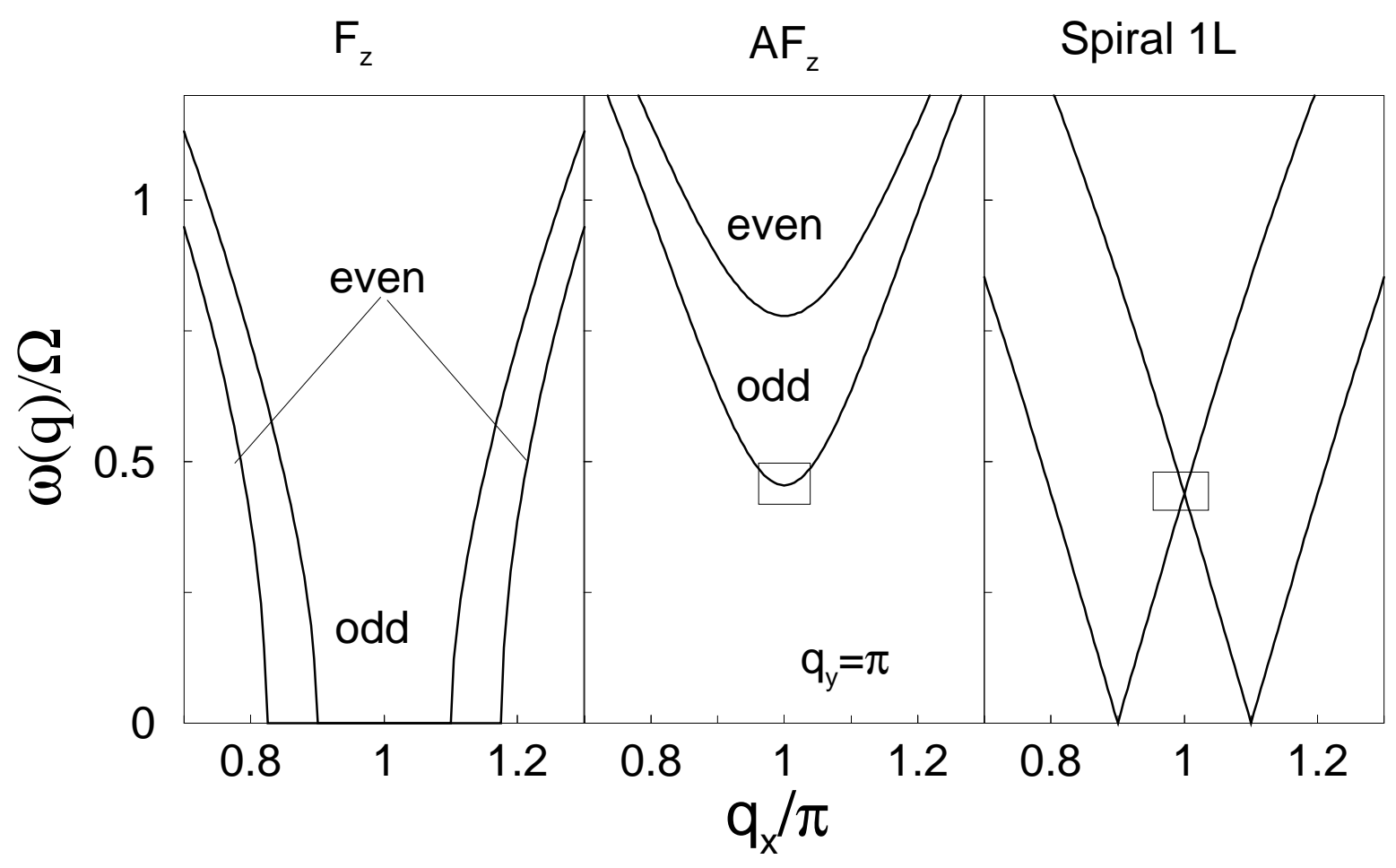

